\title{
Multiple cystic lung disease
}

\author{
Flavia Angélica Ferreira Francisco ${ }^{1}$, Arthur Soares Souza Jr², Gláucia Zanetti \\ and Edson Marchiori ${ }^{1}$
}

Affiliations: 'Dept of Radiology, Federal University of Rio de Janeiro, Rio de Janeiro, Brazil. ${ }^{2}$ Dept of Radiology, Medical School of Rio Preto and Ultra X, São José do Rio Preto, Brazil.

Correspondence: Edson Marchiori, Rua Thomaz Cameron, 438, Valparaiso, CEP 25685.120, Petrópolis, Rio de Janeiro, Brazil. E-mail: edmarchioridgmail.com

ABSTRACT Multiple cystic lung disease represents a diverse group of uncommon disorders that can present a diagnostic challenge due to the increasing number of diseases associated with this presentation. High-resolution computed tomography of the chest helps to define the morphological aspects and distribution of lung cysts, as well as associated findings. The combination of appearance upon imaging and clinical features, together with extrapulmonary manifestations, when present, permits confident and accurate diagnosis of the majority of these diseases without recourse to open-lung biopsy. The main diseases in this group that are discussed in this review are lymphangioleiomyomatosis, pulmonary Langerhans cell histiocytosis and folliculin gene-associated syndrome (Birt-Hogg-Dubé); other rare causes of cystic lung disease, including cystic metastasis of sarcoma, are also discussed. Disease progression is unpredictable, and understanding of the complications of cystic lung disease and their appearance during evolution of the disease are essential for management. Correlation of disease evolution and clinical context with chest imaging findings provides important clues for defining the underlying nature of cystic lung disease, and guides diagnostic evaluation and management.

Differential diagnosis of cystic lung disease can be achieved by correlation between clinical and HRCT findings http://ow.ly/SBTsN

\section{Introduction}

Knowledge of multiple cystic lung disease has increased in the past decade due to the widespread use of high-resolution computed tomography (HRCT). The identification of patients with asymptomatic cystic lung disease and serial scanning of symptomatic patients has furthered our understanding of cyst development, progression and prognosis $[1,2]$. A cyst is defined as any round circumscribed space that is surrounded by an epithelial or fibrous wall of variable thickness. It appears as a round parenchymal lucency or low-attenuating area that has a well-defined interface with the normal lung. Cysts in the lung usually contain air, but occasionally contain fluid or solid material [3]. They can have a variable wall thickness, but are usually thin walled $(<2 \mathrm{~mm})[2]$.

To make the correct diagnosis, use of a multidisciplinary approach that takes into consideration the patient's clinical history, physical examination findings, and radiological appearance is important. The evolution of the disease process can be assessed based on symptoms and duration, as well as comparison with previous imaging studies, when available. Acute and subacute (days to weeks) courses generally suggest infectious or inflammatory disorders, whereas a chronic course (duration $>1$ month) is more likely to be due to non-infectious infiltrative processes. In addition, the clinical context must be correlated with radiological findings. Relevant components of the clinical context include the patient's age, sex, smoking history, physical examination findings, immunocompetence, underlying diseases, prescribed $\operatorname{drug}(\mathrm{s})$ or other treatment(s), associated extrapulmonary symptoms and signs, environmental and occupational

Received: May 292015 | Accepted after revision: July 172015

Conflict of interest: None declared.

Provenance: Submitted article, peer reviewed.

Copyright OERS 2015. ERR articles are open access and distributed under the terms of the Creative Commons Attribution Non-Commercial Licence 4.0. 
exposure, recent illnesses, and relevant laboratory results $[1,2]$. Certain clinical and radiographic features may eliminate the need for surgical lung biopsy [2].

Cysts are known to occur with increasing frequency with advancing age, and are not normally expected in healthy individuals aged $<50$ years [4]. The peak incidence varies among the diseases, but typically falls in the third or fourth decade of life [1]. Sex-based predilection varies among cystic diseases [1,5-7]. Patients with different types of cystic lung disease often have similar nonspecific symptoms, such as chronic cough and shortness of breath [7]. Pneumothorax is the most common acute presentation of cystic lung disease. Spontaneous pneumothorax can be a sentinel event leading to the diagnosis of diffuse cystic lung disease $[1,2,7]$.

Imaging is thus particularly important in narrowing the differential diagnosis. Chest radiography is not a sensitive imaging modality for patients with pulmonary cysts. In these patients, chest radiographs can appear normal, or show large lung volumes and a nonspecific reticular pattern caused by the superimposition of cyst walls $[2,7,8]$. Chest HRCT is the imaging modality of choice to detect and differentiate among the various causes of cystic lung disease. Important HRCT findings that should be taken into consideration when evaluating patients with diffuse cystic lung disease include: lung volume; size, wall thickness, shape and distribution of pulmonary cysts; and associated findings, such as pulmonary nodules, septal thickening, pleural effusion, lymphadenopathy and extrathoracic abnormalities $[1,2,8]$.

In this review, we discuss the following diffuse cystic lung diseases: lymphangioleiomyomatosis (LAM), pulmonary Langerhans cell histiocytosis (PLCH), folliculin gene-associated syndrome (FLCN-S) or BirtHogg-Dubé syndrome, lymphocytic interstitial pneumonia (LIP), Pneumocystis jiroveci pneumonia (PJP), pulmonary amyloidosis, light chain deposition disease (LCDD) and metastases of sarcomas. We also comment on other rare causes of cystic disease. We highlight the epidemiology, pathology, clinical and radiological aspects, diagnostic methods, currently available treatments, and prognostic factors for these diseases. Our objective is to discuss the main aspects of each disease, to help clinicians make main diagnoses and, preferably, avoid lung biopsy.

\section{Lymphangioleiomyomatosis}

LAM is a slowly progressive lung disease that primarily affects women of reproductive age. Due to the increased detection of subclinical disease by HRCT, the mean age at the time of LAM diagnosis appears to be increasing over time, suggesting that the age distribution should be extended beyond women's reproductive years $[9,10]$. LAM can be encountered sporadically (S-LAM) or in patients with tuberous sclerosis complex (TSC). The latter is an autosomal-dominant neurocutaneous syndrome characterised by hamartoma formation in multiple organ systems, cerebral calcification, seizures, and cognitive defects [11], which can also occur in men $[12,13]$. TSC-LAM is generally thought to be 5-10 times more common than S-LAM [14].

Histologically, LAM is characterised by nodules or small clusters of smooth muscle cells (LAM cells) near cystic lesions and around terminal bronchioles, alveolar walls, pulmonary vessels, and lymphatics. Cystic changes in the pulmonary parenchyma may be the result of terminal bronchiole obstruction by LAM cells with associated air trapping, which is thought to cause progressive dilatation of the distal airspaces leading to cyst formation $[1,15]$. Degradation of the supporting architecture of the pulmonary interstitium due to metalloproteinases produced by LAM cells is considered to be an alternative or coexisting mechanism of cyst development [16].

Lymphangiogenesis has been shown to play a central pathogenetic role, whereby LAM cells invade, spread through the lymphatics and metastasise to distant sites $[17,18]$. Mutations in the tumour suppressor genes TSC1 and TSC2 are associated with the development of LAM. Mutations in the TSC2 gene arise more frequently and are associated with greater disease severity $[19,20]$. The TSC1 (encoding hamartin) and TSC2 (encoding tuberin) genes play important roles in the regulation of cell proliferation and differentiation [21].

Most patients with LAM present with pulmonary manifestations [13]. Patients with S-LAM and TSC-LAM have nonspecific symptoms, including slowly progressive dyspnoea, chest pain, cough, wheezing, and recurrent pneumothorax $[12,13]$. The mean time from symptom onset to diagnosis is typically $3-5$ years $[14,15]$. LAM is associated with an increased risk of pneumothorax, which occurs in up to $40-50 \%$ of patients at presentation and in $60-80 \%$ of patients during the course of the disease [22, 23]. The estimated recurrence rate after the first LAM episode is $75 \%$ [24].

On HRCT, cysts in LAM are typically thin walled, multiple, well circumscribed, and distributed diffusely throughout the lungs with normal intervening lung, without lobar predominance (fig. 1). Cysts are round and are usually $2-5 \mathrm{~mm}$ in diameter, but are occasionally as large as $30 \mathrm{~mm}$ [25]. As the disease progresses to the advanced stages, some cysts may coalesce into larger bodies with near-complete replacement of the lung; eventually, the lung may appear very similar to that observed in end-stage emphysema $[14,25]$. Generally, the lung parenchyma outside the cysts appears normal, but patchy 
a)

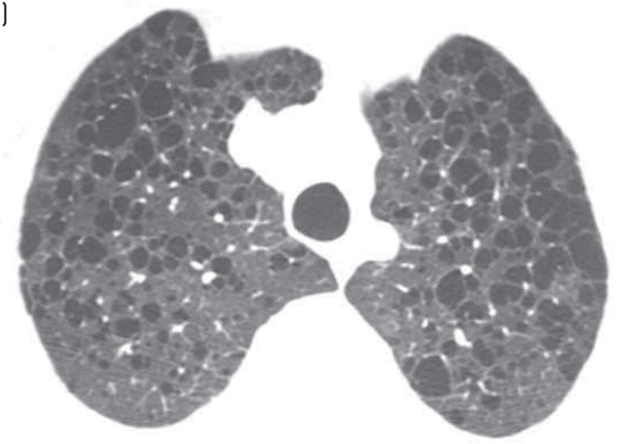

b)

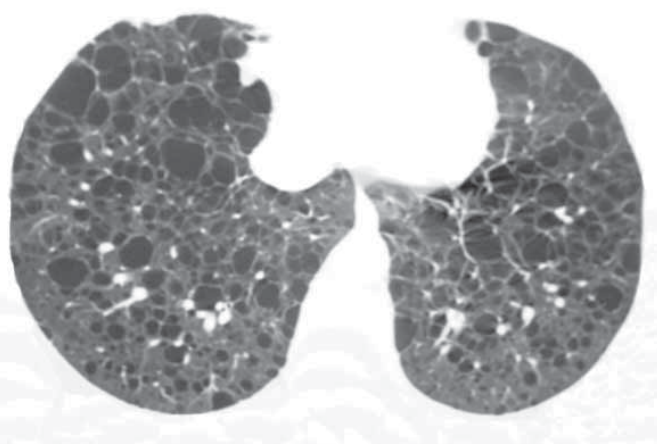

FIGURE 1 A 38-year-old woman with lymphangioleiomyomatosis. High-resolution computed tomography scans of al upper and b) lower lobes show multiple thin-walled cysts distributed symmetrically throughout both lungs, with normal intervening lung parenchyma.

ground-glass opacities can occur, presumably from recurrent haemorrhage and/or relatively diffuse proliferation of immature smooth muscle cells. Areas of air trapping can be found on the expiratory portion of the computed tomography (CT) scan [26]. Nodules are rarely seen in LAM. Nodular lesions, solid or with a ground-glass appearance, are more commonly seen in patients with TSC $[27,28]$. Nodules range in size from 1 to $10 \mathrm{~mm}$, and are usually upper-lobe predominant, tiny and peripheral. These nodules represent a benign process, multifocal micronodular pneumocyte hyperplasia, which occurs in men and women with TSC [29].

Lymphatic abnormality is common in S-LAM due to lymphatic obstruction, which can lead to interstitial septal thickening, chylous pericardial or pleural effusion, thoracic and abdominal lymphadenopathy, thoracic duct enlargement, diffusely thickened mediastinum with decreased density, and lymphangioleiomyoma in the thorax and abdomen $[26,27]$.

Non-lymphatic extrathoracic features of LAM include hepatic and renal angiomyolipoma, as well as meningioma. Renal angiomyolipoma is the most common and characteristic extrathoracic manifestation, seen in up to half of cases [30]. On CT, renal angiomyolipomas consist of areas of fatty density intermixed with denser areas and renal parenchyma with a normal appearance. The tumours are highly vascular, with the blood supply usually originating from the renal arteries. They range in diameter from $1 \mathrm{~mm}$ to more than $20 \mathrm{~cm}$ and may completely disrupt the normal kidney architecture [31].

Although biopsy is the gold standard for the diagnosis of LAM, some studies have shown that a confident CT diagnosis can be made in $72-79 \%$ of cases $[32,33]$. The European Respiratory Society LAM task force recently published diagnostic guidelines [34]. The diagnosis of LAM is classified as definite, probable or possible. The HRCT appearance of the lungs is classified as characteristic or compatible with this diagnosis. A characteristic HRCT appearance requires multiple $(>10)$ thin-walled cysts with normal or increased lung volumes, with no other significant interstitial abnormality. Compatible HRCT findings are defined by the appearance of fewer $(>2$ but $<10)$ typical cysts [34].

Vascular endothelial growth factor D (VEGF-D), a lymphangiogenic growth factor, is a useful marker for the diagnosis of LAM in patients with cystic lung disease and may also have prognostic significance. Patients with LAM demonstrate higher serum concentrations of VEGF-D compared with the healthy normal population. VEGF-D levels $>800 \mathrm{pg} \cdot \mathrm{mL}^{-1}$ and the presence of pulmonary cysts on imaging are specific for S-LAM and identify LAM in females with TSC [35]. This biomarker is particularly helpful in patients who present with isolated pulmonary cystic lung disease, for whom open-lung biopsy is often recommended to confirm the diagnosis of LAM [36].

LAM is a progressive lung disease with a poor long-term prognosis. Several therapeutic options have been studied based on the relationship between hormonal stimulation and worsening/recurrence of symptoms, with controversial results. As LAM is a disease of females and is thought to be accelerated by oestrogen, this hormone should be avoided [22, 37, 38]. Promising new treatment strategies have been introduced recently due to discovery of the genetic and molecular mechanisms of LAM. Mutations in the TSC genes lead to the activation of mammalian target of rapamycin kinase (mTOR), resulting in proliferation of LAM cells. The mTOR inhibitor sirolimus (rapamycin) appears to be the most effective drug in this treatment approach [39]. Treatment with sirolimus can stop the decline in lung function and improve quality of life [34, 40]. Lung transplantation is an option for patients with severe impairment due to progressive LAM [41], and is the only current curative therapy for these patients. However, recurrence of 
LAM in the transplanted lung has been observed [42]. The clinical course of LAM depends on the age at presentation. Patients diagnosed at younger ages tend to have more aggressive courses. Presentation following menopause tends to be more benign, with longer survival $[22,34]$.

\section{Pulmonary Langerhans cell histiocytosis}

PLCH is diagnosed predominantly in younger adults, during the third or fourth decade of life, but reports show an age range of 1-69 years [1,43]. PLCH has no sex-based predominance [44, 45]. The most striking epidemiological characteristic of adult PLCH is that $90-100 \%$ of patients are smokers, often smoking $>20$ cigarettes per day [45]. Thus, PLCH is a disease of current and former smokers. No other epidemiological factor associated with PLCH has been identified [46], and no genetic risk factor for PLCH development has been recognised [7].

Histologically, PLCH is characterised by peribronchiolar infiltration by Langerhans cells and formation of granulomas, leading to stellate nodule formation. These stellate nodules can later cavitate, resulting in bronchial dilatation and formation of thin- and then thick-walled cysts and cavities [34, 47]. These cysts may coalesce, forming bizarre shapes [48]. Traction emphysema also contributes to the cystic appearance of advanced lesions [49]. The cystic pattern does not currently have a conclusive radiological-pathological correlation. Cysts may develop in and around PLCH lesions, and are thought to represent airway lumina that enlarge as a result of bronchiole wall inflammation, cavitary granulomas (probably corresponding to cellular lesions surrounding dilated bronchioles), coalescence of adjacent affected airways, and paracicatricial air-space enlargement due to fibrous lesions lacking Langerhans cells $[49,50]$.

The presentation of PLCH is pleomorphic. Despite diffuse lung involvement, symptoms are minor or absent in $\sim 25 \%$ of patients $[44,51]$. The main respiratory symptoms are dry cough and dyspnoea on exertion, which can be associated with nonspecific constitutional manifestations such as asthenia, fever, night sweats and weight loss $[45,46]$. Spontaneous pneumothorax (responsible for chest pain) leads to the diagnosis in $\sim 10-20 \%$ of cases [46]. It appears to occur more commonly in young males, at any time during the course of the disease, and may be bilateral and/or recurrent $[44,47]$. PLCH in adults is usually a single-system disease. Bone lesions $(<20 \%$ of patients), diabetes insipidus with polyuria and polydipsia resulting from infiltration of the posterior pituitary (5\% of patients), and skin lesions are the most common extrapulmonary manifestations $[44,45]$.

A well-recognised sequence of radiographic events occurs in patients with PLCH, and CT findings reflect the temporal heterogeneity of the disease. On HRCT, the early manifestation of PLCH is characterised by a predominantly nodular pattern; a predominance of thin-walled cysts is typically seen in later phases of the disease. In most patients, the main imaging feature is a combination of nodular and cystic lesions $[46,50]$. Nodules are the most common radiographic manifestation in the early phase of the disease. They are typically $1-10 \mathrm{~mm}$ in diameter, but may be as large as $1.5-2 \mathrm{~cm}$; they are usually bilateral and symmetric in distribution. Their distribution is typically centrilobular, but they are often peribronchial or peribronchiolar, which may correlate with the bronchiolocentric orientation of the lesions [49, 50]. The margins are characteristically irregular, especially when cystic or fibrotic changes are present in the adjacent lung, but smooth margins have also been described [50].

Cystic lesions are the most common HRCT feature of PLCH. They usually measure $<10 \mathrm{~mm}$ in diameter, but may be as large as $20 \mathrm{~mm}[49,50]$. They may manifest as round or ovoid cystic spaces or exhibit bizarre configurations with two-lobed, clover-leaf, septated and branching morphologies, resembling bronchiectasis. These appearances may result from coalescence of adjacent cysts or reflect the peribronchiolar distribution of PLCH lesions [50]. Cyst walls may be thin and barely perceptible or may appear thick, irregular, and nodular on HRCT scans, depending on the degree of nodule cavitation [46, 50]. Different cyst appearances may coexist (fig. 2) [50]. Late in the course of PLCH, patients may develop secondary pulmonary arterial hypertension, because pulmonary vascular lesions tend to predominate in the venules and may produce a picture reminiscent of pulmonary veno-occlusive disease [52].

Nodules and cysts follow an apicobasal gradient of severity, being larger and more numerous in the upper lobes than in the lower lung zones. This differential severity occurs because PLCH is a smoking-related disease, and more ventilation (increased cigarette smoke) occurs in the upper than in the lower lobes. The costophrenic angles, lung bases, and anterior tips of the right middle lobe and lingula are typically spared $[48,50]$.

The diagnosis of PLCH is highly likely in a young smoker with evidence of pulmonary nodules and cysts showing an upper lobe distribution with sparing of the costophrenic sulci. In cases of initial diagnostic uncertainty, bronchoscopy with bronchoalveolar lavage (BAL) and biopsy may yield diagnostic results [53]. The definitive diagnosis of PLCH requires identification of Langerhans cell granulomas, which is usually achieved by surgical lung biopsy at a site selected using chest HRCT [46]. 

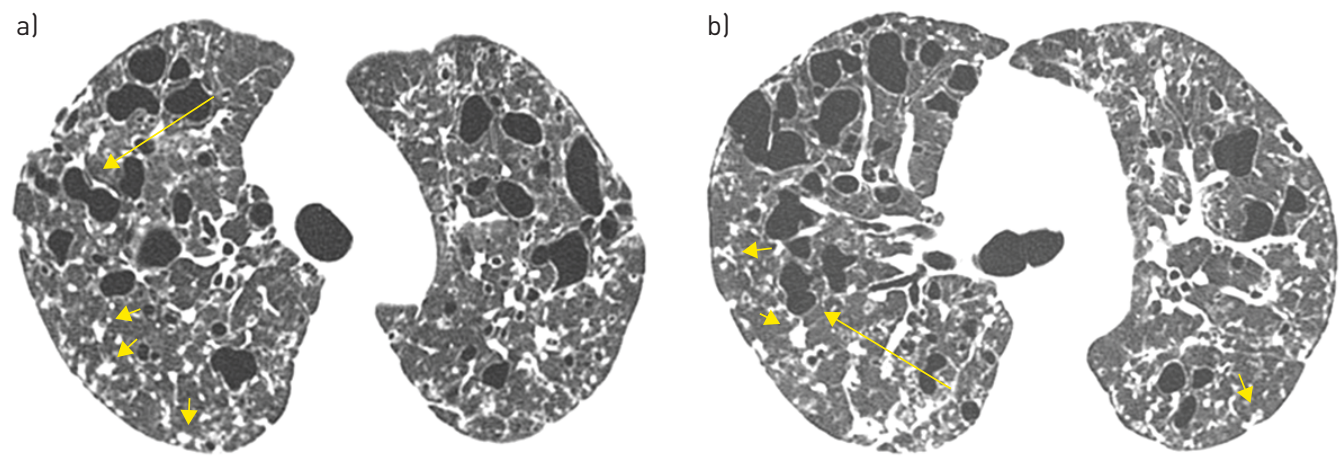

FIGURE 2 A 27-year-old man with pulmonary Langerhans cell histiocytosis. High-resolution computed tomography scans of a) upper and b) middle lung regions show multiple thin-walled cysts, some with irregular shapes (arrows), in both lungs. Multiple small nodules (short arrows) are also present.

Complete remission of PLCH may occur on smoking cessation; in a significant number of patients, cessation is the only therapeutic intervention needed. As many patients with PLCH recover spontaneously or remain stable without treatment, the effectiveness of the various treatments used for this condition is difficult to assess. In practice, no effective treatment is available $[46,50]$. Pneumothorax requires drainage, pleurodesis may also be needed, and pleurectomy is best avoided in young patients, who may later be candidates for lung transplantation [51]. Cladribine (2-chlorodeoxyadenosine), a synthetic purine analogue chemotherapeutic agent, has been reported to be effective in several cases of progressive PLCH, with imaging-detected improvement including a reduction in the size and number of nodules [54,55]. A number of patients with very severe respiratory failure or major pulmonary hypertension have been treated with lung transplantation, with results similar to those found in patients with other patterns of diffuse infiltrating lung disease [56]. Death may result from respiratory insufficiency and/or pulmonary hypertension. The mortality rate is difficult to evaluate because patients with minor symptoms are frequently lost to follow-up [51]. However, the median duration of survival from the time of diagnosis is 12.5 years [51]. Furthermore, PLCH may recur following radiological regression of nodular lung abnormalities up to 7.5 years after the patient's initial presentation, even in individuals who have stopped smoking [57].

\section{Folliculin gene-associated syndrome (Birt-Hogg-Dubé syndrome)}

FLCN-S, a more appropriate terminology than Birt-Hogg-Dubé syndrome [58], is an autosomal-dominant genodermatosis that manifests as a multisystemic disorder, with most clinical findings observed in the skin, lungs and kidneys [59]. However, some patients with FLCN-S have only multiple pulmonary cysts, with no other feature [60]. The disease typically manifests in the third or fourth decade of life, but FLCN-S has been described in patients aged $20-85$ years. It has no gender predilection [61].

Histopathologically, the inner surfaces of cysts are lined by epithelial cells, sometimes with a predominance of type II pneumocyte-like cuboidal cells. No evidence of neoplastic proliferation, inflammation, fibrosis or atypical morphology is found. The cysts occasionally contain internal septa consisting of alveolar walls, and a pattern known as "alveoli within an alveolus" is formed when a cyst wall is partially enveloped by another wall. Some cysts have veins protruding into the cystic space. These characteristic findings are important microscopic clues for the pathological diagnosis of FLCN-S [61, 62]. FLCN-S is a tumour suppressor syndrome resulting from mutations in the gene encoding the protein folliculin, leading to abnormal mesodermal development. Folliculin is a tumour-suppressor protein that is highly expressed in the stromal cells and type I pneumocytes of the lungs, as well as in the skin and kidneys [63]. This aetiology could explain the propensity for lung cyst formation in patients with FLCN-S [64], but the exact mechanism for the development of pulmonary cysts in this syndrome remains unclear [65].

Affected patients may be asymptomatic, but the most common presentation is skin papules. The typical skin manifestations of FLCN-S include facial papules (fibrofolliculomas and trichodiscomas) and skin tags (acrochorda) on the head, neck and upper trunk $[63,66]$. The majority of patients with pulmonary cysts have facial fibrofolliculomas, although skin lesions may be entirely absent [67]. Renal findings can range from benign cysts to malignant tumours, and are noted at an earlier age (mean 50.7 years) in these patients than in the general population. In more than half of these cases, the tumours are bilateral and multifocal; they can range from benign oncocytomas to malignant renal carcinomas $[68,69]$. 
The most common presentation of pulmonary involvement with FLCN-S is spontaneous pneumothorax [61], estimated to occur in $\sim 35 \%$ of patients, representing a 50 -fold increase in the rate of development of this condition [69]. A family history of pneumothorax is present in 35\% of patients [70], and pneumothorax recurs in the majority (75\%) of patients [71]. In FLCN-S, cysts are bilateral, range in number from a few to more than 100, and range in size from a few millimetres to several centimetres (maximum diameter $2-8 \mathrm{~cm}$ ). The cysts are thin walled and sometimes septated. They can be round, oval, lentiform, lobulated or irregularly shaped, and are generally surrounded by perceptible thin walls. Large cysts, particularly those in the lower lungs, have a lobulated, multi-septated appearance [65, 72]. Predominant distribution of cysts in the lower lung zone is a characteristic finding [64, 65, 72]. The remaining lung parenchyma is generally normal (fig. 3) [64].

The typical presentation of FLCN-S involves skin lesions and a history of recurrent and familial pneumothorax. The majority of patients with FLCN-S have pulmonary cysts, which may develop earlier than skin and renal manifestations. FLCN-S may manifest only as relapsing pneumothorax and multiple lung cysts, occasionally with no skin or renal lesions [60]. In this context, the detection of pulmonary cysts may be useful for early diagnosis of this syndrome, and chest CT may play an important role [65, 72].

The definitive diagnosis of FLCN-S should ideally rely on the finding of a mutation in the FLCN gene and the presence of multiple lung cysts and/or characteristic skin lesions and/or malignant kidney tumour [58]. Patients suspected of having FLCN-S may be offered genetic testing for mutations of the FLCN gene, which not only confirms the diagnosis, but also facilitates pre-symptomatic testing of at-risk family members $[48,66]$. At present, the management of cystic lung disease associated with FLCN-S focuses on the management of pneumothorax, typically with tube thoracostomy followed by pleurodesis to prevent recurrence [61]. No specific therapy for this cystic lung disease is currently available. Whether mTOR inhibition has a role in the treatment of patients with FLCN-S and progressive cystic lung disease is currently unclear [67]. The prognosis is based on comorbidities, such as renal cell carcinoma or pneumothorax, rather than on the lung cysts. In FLCN-S, lung disease progresses slowly and appears to be more severe in smokers than in nonsmokers $[61,67]$.

\section{Lymphocytic interstitial pneumonia}

LIP is a benign lymphoproliferative disorder that commonly affects middle-aged women. It is most commonly seen in association with various underlying disorders, including HIV infection, connective tissue diseases such as Sjögren's syndrome, Hashimoto's thyroiditis, and systemic lupus erythematosus [73,
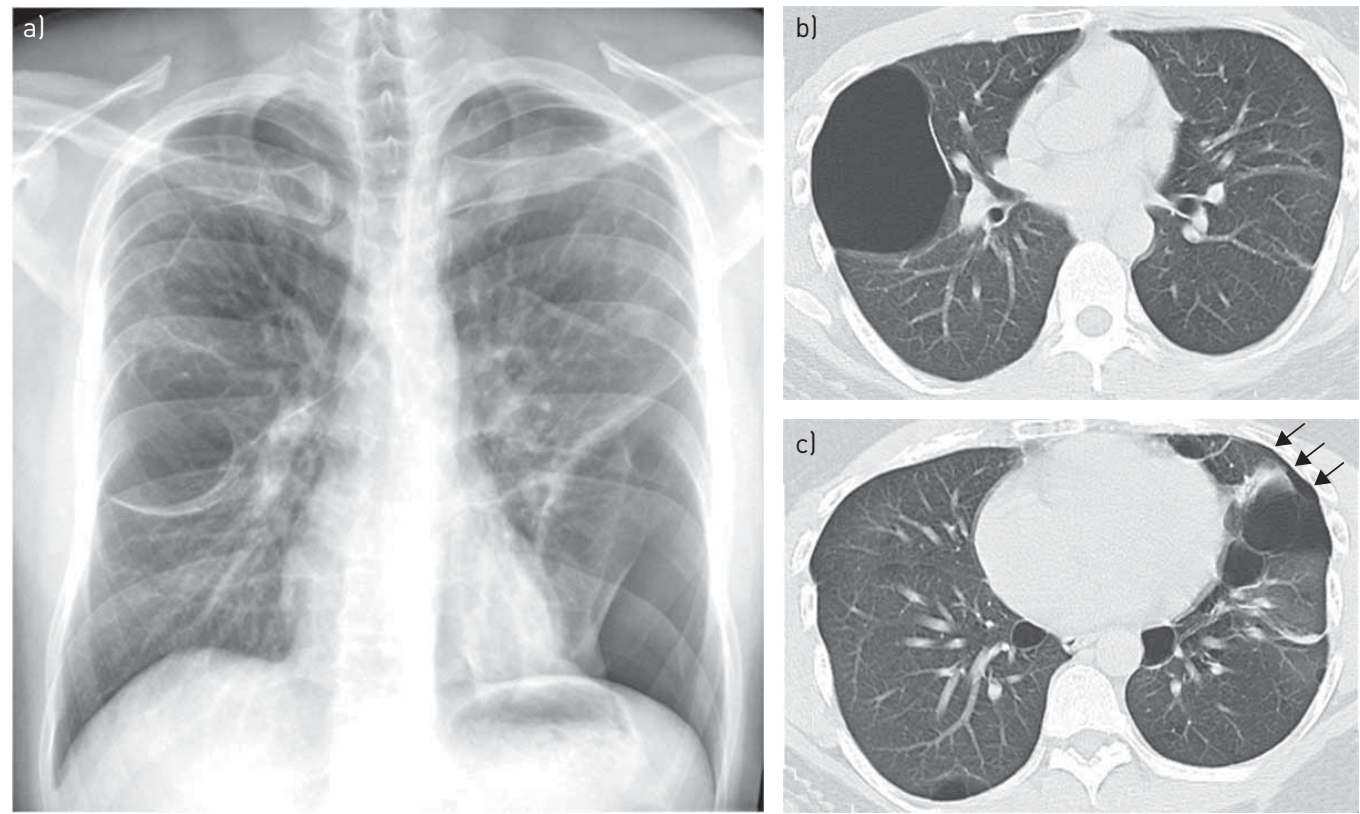

FIGURE 3 A 33-year-old man with folliculin gene-associated syndrome (Birt-Hogg-Dubé syndrome). a) A chest radiograph shows bilateral bullous formation with left basal pneumothorax. b, c) High-resolution computed tomography performed 1 week later demonstrates thin-walled cysts of varying sizes in both lungs, some with a lentiform shape, with characteristic lower lung zone distribution. A small residual left pneumothorax (arrows) is also present. 
74]. It can also occur alone as a form of idiopathic interstitial pneumonia [75]. LIP in adults that is not associated with HIV infection usually occurs in women in the fourth or fifth decade of life [6].

LIP is characterised by diffuse interstitial inflammatory infiltrate composed of lymphocytes, plasma cells and occasional histiocytes [73]. This pattern is most characteristically present in the areas surrounding lymphatic channels, such as the alveolar septa, interstitial septa, peribronchovascular regions and subpleural lung. Cyst formation in LIP appears to arise from peribronchiolar lymphoid infiltration resulting in bronchiolar stenosis or occlusion, leading to air trapping and distal airway dilatation [73, 74].

The clinical presentation of LIP is nonspecific, and includes dyspnoea, cough, fever and weight loss in the majority of patients. The cystic changes may predispose some patients to recurrent pneumothorax [48]. Additional clinical features that reflect the underlying systemic disease may be noted [74].

HRCT usually demonstrates a combination of ground-glass opacification, consolidation, poorly defined centrilobular nodules, small subpleural nodules, interlobular septal thickening, thickening of the bronchovascular bundles and scattered cysts (fig. 4). In the acute phase, diffuse or multifocal ground-glass opacities representing areas of alveolar septal infiltration are seen $[6,74,76]$. Lung cysts are present in up to $80 \%$ of patients; they are usually few in number but are distributed diffusely in both lungs, although they are often subpleural and peribronchovascular. Cysts in LIP are typically large, but generally measure $<3 \mathrm{~cm}$ in diameter; they vary in shape and may be the sole manifestation of this disease [74, 76]. In most cases, follow-up CT reveals the resolution of ground-glass opacity; cysts are the only residual finding in more chronic cases $[48,74]$.

The diagnosis of LIP should be considered in a patient with lung cysts and an immunological abnormality. The appearance of a few scattered cysts in a patient with Sjögren's syndrome is very likely to be due to LIP [77].

Treatment of LIP depends in part on the nature of the underlying disorder and is directed at the underlying systemic disease. LIP is considered to be a steroid-responsive disease, but the response to therapy is unpredictable. Cysts persist after therapy $[6,76] . \sim 5 \%$ of patients with LIP develop lymphoma, but whether this is a consequence of LIP or whether LIP was a manifestation of underlying lymphoma is unclear [6]. Prognosis of these patients is variable and is influenced by the course of any underlying systemic disease [73]. The disease can resolve (spontaneously or with corticosteroid treatment), remain stable over a period of years, or progress over time, leading to pulmonary fibrosis and eventual respiratory failure $[6,68] . \sim 33-50 \%$ of patients die within 5 years of diagnosis [6].

\section{Pneumocystis jiroveci pneumonia}

PJP, a fungal infection, is seen almost exclusively in immunosuppressed patients, especially in those with AIDS who have CD4 counts $<200$ cells $\mathrm{mm}^{-3}$. The incidence of PJP has decreased dramatically with the advent of antiretroviral therapy [78]. Patients with PJP have nonspecific symptoms, such as nonproductive cough, low-grade fever, dyspnoea and spontaneous pneumothorax [79]. The symptoms can be insidious, but may progress to respiratory failure and even death, often following a rapid, fulminant course, if left untreated $[78,79]$. Serum lactate dehydrogenase levels are elevated $\left(>220 \mathrm{U} \cdot \mathrm{L}^{-1}\right)$ in $90 \%$ of patients [79]. BAL has a diagnostic yield exceeding $90 \%$, but it is less sensitive in patients receiving aerosolised pentamidine. Transbronchial biopsy is recommended for such patients [79].
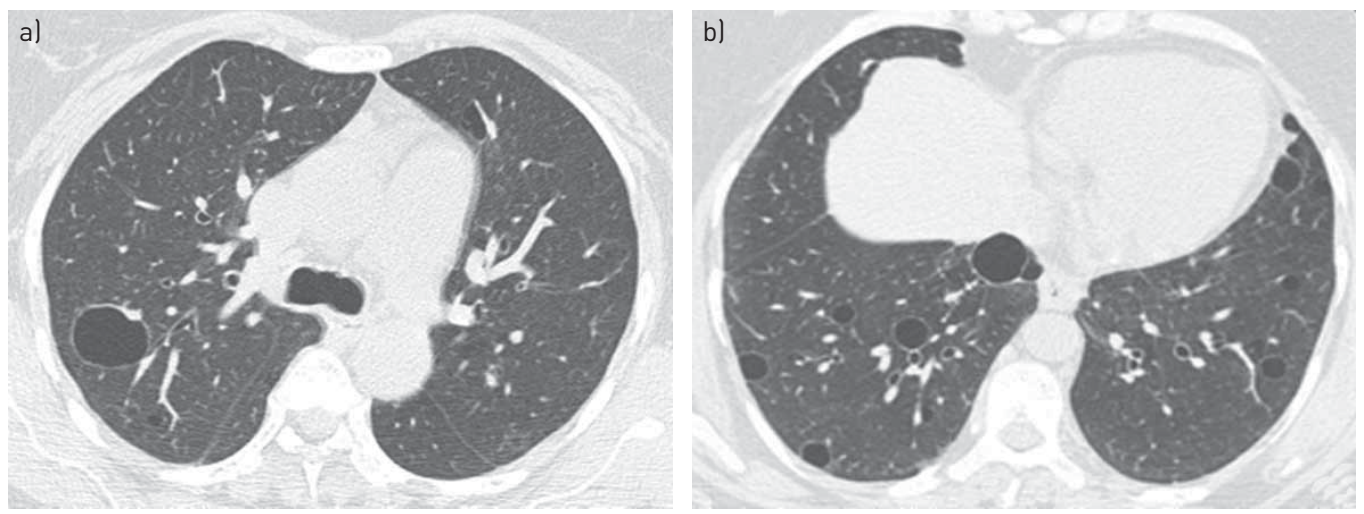

FIGURE 4 A 43-year-old woman with lymphocytic interstitial pneumonia in association with Sjögren's syndrome. High-resolution computed tomography scans of al middle and b) lower lung regions show bilateral thin-walled cysts. 
The radiographic manifestations of PJP vary widely, ranging from totally normal chest radiographic findings to widespread, bilateral and symmetric opacities. On HRCT, ground-glass opacities representing acute pneumonitis are the dominant feature. The pattern of these opacities is often bilateral, multifocal and mainly symmetric, distributed in the central portions of the lungs. Opacities can predominate in the upper lung zones in patients receiving prophylactic therapy for this infection $[79,80]$. More focal areas of consolidation are also common [81]. Patients with PJP develop small thin- or thick-walled intrapulmonary cysts within the ground-glass opacities (fig. 5) [80, 81]. These cysts tend to occur after multiple infections, often have an apical predominance and may lead to pneumothoraces, which portend a poor outcome [82]. Other less-frequent manifestations include a reticular or reticulonodular pattern and pulmonary nodules. Pleural effusion is almost always absent, and its presence should raise the possibility of an alternate diagnosis [2]. 10-34\% of HIV-positive patients with PJP develop post-infectious pulmonary fibrosis [83].

\section{Amyloidosis}

Amyloidosis refers to systemic or organ-limited disease resulting from extracellular deposition of insoluble fibrillar protein (amyloid) in tissue. This disease most commonly has systemic involvement, but a small percentage of patients present with localised disease [82]. Amyloid deposition in the respiratory tract has a broad spectrum of manifestations, including tracheobronchial disease, parenchymal nodules and infiltrates, pleural disease, and lymphadenopathy [84, 85]. Pulmonary involvement in amyloidosis is usually associated with light chain amyloidosis [79]. The diagnosis is confirmed by tissue biopsy demonstrating amorphous material that stains positively with Congo red [85].

Pulmonary amyloidosis can also present with cystic lung disease $[84,86]$. In these cases, scattered cysts of varying sizes are seen, sometimes in association with Sjögren's syndrome or mucosa-associated lymphoid tissue lymphoma [87]. Pulmonary cyst formation in amyloidosis is thought to involve multiple mechanisms, including: airway narrowing due to infiltration of the small airways by amyloid and inflammatory cells, with subsequent expansion and destruction of the alveoli from a ball-valve effect; increased fragility caused by amyloid deposition with disruption of the alveolar structures; and ischaemia related to vascular amyloid deposits and reduced blood flow [88]. On HRCT, the cysts demonstrate thin walls and are usually located along the periphery of the lung parenchyma, with no apicobasal gradient [84, 87]. Multiple small lung nodules (with or without calcification) may also be present, commonly in association with Sjögren's syndrome, and may abut the walls of the parenchymal cysts [87]. Other CT findings include interlobular septal thickening, honeycombing, ground-glass opacities, circumferential thickening of the tracheal wall and lymphadenopathy $[84,87]$.

Pulmonary amyloidosis associated with systemic disease has a poor long-term outcome after diagnosis, although patients with isolated pulmonary amyloidosis have a benign clinical course with a good prognosis [84].

\section{Light chain deposition disease}

LCDD is characterised by the systemic accumulation of immunoglobulin light chains. It is usually seen in middle-aged individuals, with no sex predilection. $\sim 75 \%$ of patients have an underlying plasma cell dyscrasia, such as multiple myeloma, Waldenström macroglobulinaemia and B-cell lymphomas [5, 48]; most other cases are also associated with autoimmune conditions, and a few cases are idiopathic [5]. LCDD is a multisystem disease, most commonly involving the kidney, followed by the heart and liver. Cases involving the kidney result in proteinuria, with or without nephrotic syndrome, and renal failure. Lung involvement has been described in only a few cases $[1,5]$.

a)

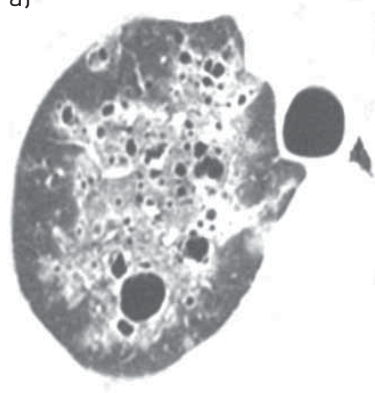

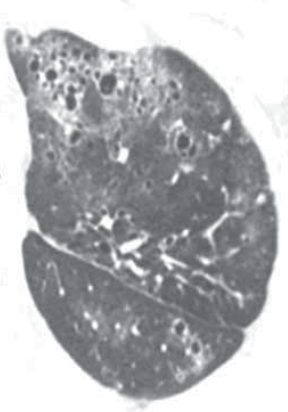

b)

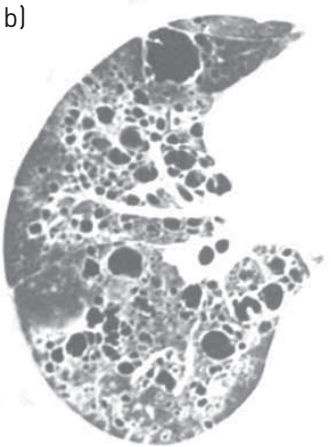

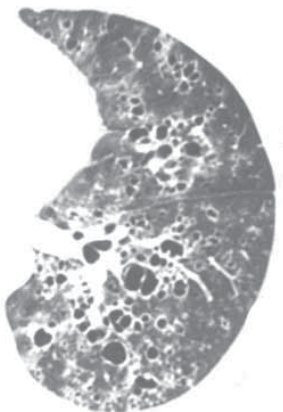

FIGURE 5 A 37-year-old man with Pneumocystis jiroveci pneumonia. High-resolution computed tomography scans of al upper and b) lower lobes demonstrate thin-walled cysts associated with ground-glass opacities distributed throughout both lungs. 
LCDD is also a monoclonal plasma cell proliferative disorder that results from tissue deposition of light chain fragments that do not form amyloid fibrils and thus do not stain positively with Congo red. This indication that the material is not amyloid leads to the diagnosis of LCDD [5].

Pathologically, the disease is characterised by the deposition of extracellular, amorphous, eosinophilic material along the perilymphatic tissues and surrounding bronchovascular bundles, involving the interlobular septa or in a more alveolar location $[5,89]$. The diagnosis of LCDD may also be confirmed by the presence of a monoclonal free light chain, especially chain $\kappa$ in the serum or urine $[5,90]$.

The most common HRCT findings include cysts and nodules. Cysts are generally thin walled, spherical, and diffusely distributed. They can progress in number and size, with some cysts coalescing to form irregular shapes. Mean cyst size is $10 \mathrm{~mm}$ (range 4-15 mm) [5, 89]. Nodules can be solitary or multiple and bilateral, and range in size from $2 \mathrm{~mm}$ to $5 \mathrm{~cm}[90,91]$.

Haematological malignancy, particularly myeloma, is found in the majority of patients with LCDD. Pulmonary LCDD should be considered in any patient with a history of multiple myeloma or lymphoproliferative disorder presenting with cystic lung disease [91]. The prognostic factors are advanced age, associated multiple myeloma and extra-renal light chain deposition. LCDD appears to be indolent, but respiratory failure with lung transplantation has been reported [5, 89]. Management remains controversial, although autologous stem cell transplantation and immunomodulatory drugs have been employed with variable results $[5,91]$.

\section{Neoplastic disease}

Rarely, neoplastic processes cause multiple cystic lung lesions. This presentation has been described with several types of metastatic cancer, including metastatic sarcomas. Pneumothorax may be the presenting manifestation of an extrapulmonary sarcoma with cystic metastasis, especially scalp angiosarcoma [92]. Proposed mechanisms for the genesis of cystic metastatic lung lesions are excavation of a metastatic nodule, infiltration of tumour cells into the walls of air spaces with the ball-valve effect, and the development of blood-filled cystic spaces that are characteristic of angiosarcoma [93]. Direct involvement of the tumour within the pleura, or extension of cavitary tumour lesions into the pleural space, are cited most commonly as the mechanism leading to the development of spontaneous pneumothorax [93]. Chemotherapeutic agents have been implicated in spontaneous pneumothorax, probably due to necrosis of peripheral pulmonary nodules in response to therapy [92].

On CT, these metastases appear as solid nodules, thin-walled cystic lesions, and air-fluid levels within cysts indicating haemorrhage [93]. As with other metastatic lesions, cystic metastases tend to have different sizes and a basilar predominance [2].

\section{Other multiple cystic lung diseases}

Depending on the clinical context, several other disorders must be considered in the differential diagnosis of diffuse cystic lung disease. Desquamative interstitial pneumonia is a type of idiopathic interstitial pneumonia, but is probably a form of smoking-related interstitial lung disease [47]. The most common HRCT findings are ground-glass opacities, but some patients also exhibit small cystic lesions [94]. Cysts can be found in a minority of patients with hypersensitivity pneumonitis. They are usually few in number, range from $3 \mathrm{~mm}$ to $25 \mathrm{~mm}$ in diameter, and have a random distribution [95]. Bronchiolitis is usually characterised by small centrilobular nodules, the "tree-in-bud" pattern, bronchiolar wall thickening, bronchiolectasis, and a mosaic attenuation pattern. Cysts are uncommon and can occur in follicular bronchiolitis and, rarely, in patients with constrictive bronchiolitis [96]. Several hereditary syndromes other than TSC, including Marfan syndrome, neurofibromatosis 1, Ehlers-Danlos syndrome and Proteus syndrome, have been associated with cystic lung disease. In all of these conditions, the number of pulmonary cysts is limited [97-101]. Regarding infectious causes, multiple thin-walled cysts may form as the primary infection resolves in cases of chronic pulmonary paracoccidioidomycosis and coccidioidomycosis [102-104]. Tracheobronchial papillomatosis rarely affects the pulmonary parenchyma. When it does, tomographic findings include cystic lucencies and pulmonary nodules, as well as papillomas in the airway [105-107].

\section{Pitfalls}

Emphysematous changes present pitfalls that can lead to confusion about cysts. In contrast to true cysts, however, cyst-like lucencies caused by the destruction of lung parenchyma in emphysema do not have walls [108]. Nevertheless, emphysema can be accompanied by multiple cysts, such as bullae, or can present in association with PLCH [2]. A bulla is defined as a sharply demarcated area of emphysema with a diameter $\geqslant 1 \mathrm{~cm}$ and wall thickness $\leqslant 1 \mathrm{~mm}$ [3]. Bullae are located in the subpleural region, rather than within the lung parenchyma, and are manifestations of paraseptal emphysema, although they can also occur in centrilobular emphysema [108]. 
Bronchiectasis is defined as localised dilatation of the bronchial tree. Cystic bronchiectasis can also be mistaken for cysts; multiplanar reformations can be helpful in distinguishing this disease, which is characterised by continuity of a bronchus [2]. Honeycombing, found in patients with end-stage pulmonary fibrosis, appears as multiple rows of air-filled spaces clustered in the subpleural region, predominantly in the lower lobes and typically $<1 \mathrm{~cm}$ in diameter [3]. Pneumatoceles manifest on CT as scattered, thin-walled, gas-filled cysts interspersed with normal lung in areas previously affected by pneumonia, especially that caused by Staphylococcus aureus or trauma $[2,109]$. Finally, a cavity is differentiated from a lung cyst by the presence of a thick wall (generally $>4 \mathrm{~mm}$ ). Cavitary lesions in the lung parenchyma can be caused by primary bronchogenic carcinoma, lung metastases, vasculitides and various infectious aetiologies [82, 87].

\section{Conclusion}

Multiple cystic lung diseases are uncommon and consist of multiple entities associated with different pathological processes. In recent years, significant advances have been achieved in the understanding of these diseases and their diverse imaging aspects. Differentiating among these diseases can be achieved by consideration of the clinical context, as well as cyst characteristics on HRCT and associated radiological aspects, including extrapulmonary findings. Optimal management of these patients requires a correct diagnosis that can be reached by thoughtful analysis of these presenting features, avoiding lung biopsy in most cases.

\section{References}

$1 \quad$ Ryu JH, Tian X, Baqir M, et al. Diffuse cystic lung diseases. Front Med 2013; 7: 316-327.

2 Cantin L, Bankier AA, Eisenberg RL. Multiple cystlike lung lesions in the adult. AJR Am J Roentgenol 2010; 194: W1-W11.

3 Hansell DM, Bankier AA, MacMahon H, et al. Fleischner Society: glossary of terms for thoracic imaging. Radiology 2008; 246: 697-722.

4 Copley SJ, Wells AU, Hawtin KE, et al. Lung morphology in the elderly: comparative CT study of subjects over 75 years old versus those under 55 years old. Radiology 2009; 251: 566-573.

5 Colombat M, Stern M, Groussard O, et al. Pulmonary cystic disorder related to light chain deposition disease. Am J Respir Crit Care Med 2006; 173: 777-780.

6 Cha SI, Fessler MB, Cool CD, et al. Lymphoid interstitial pneumonia: clinical features, associations and prognosis. Eur Respir J 2006; 28: 364-369.

7 Trotman-Dickenson B. Cystic lung disease: achieving a radiologic diagnosis. Eur J Radiol 2014; 83: 39-46.

8 Lee KH, Lee JS, Lynch DA, et al. The radiologic differential diagnosis of diffuse lung diseases characterized by multiple cysts or cavities. J Comput Assist Tomogr 2002; 26: 5-12.

9 Cohen MM, Pollock-BarZiv S, Johnson SR. Emerging clinical picture of lymphangioleiomyomatosis. Thorax 2005; 60: 875-879.

10 Hohman DW, Noghrehkar D, Ratnayake S. Lymphangioleiomyomatosis: a review. Eur J Intern Med 2008; 19: 319-324.

11 Sparagana SP, Roach ES. Tuberous sclerosis complex. Curr Opin Neurol 2000; 13: 115-119.

12 Meraj R, Wikenheiser-Brokamp KA, Young LR, et al. Lymphangioleiomyomatosis: new concepts in pathogenesis, diagnosis, and treatment. Semin Respir Crit Care Med 2012; 33: 486-497.

13 Ryu JH, Moss J, Beck GJ, et al. The NHLBI lymphangioleiomyomatosis registry: characteristics of 230 patients at enrollment. Am J Respir Crit Care Med 2006; 173: 105-111.

14 McCormack FX. Lymphangioleiomyomatosis: a clinical update. Chest 2008; 133: 507-516.

15 Juvet SC, McCormack FX, Kwiatkowski DJ, et al. Molecular pathogenesis of lymphangioleiomyomatosis: lessons learned from orphans. Am J Respir Cell Mol Biol 2007; 36: 398-408.

16 Matsui K, Takeda K, Yu ZX, et al. Role for activation of matrix metalloproteinases in the pathogenesis of pulmonary lymphangioleiomyomatosis. Arch Pathol Lab Med 2000; 124: 267-275.

17 Finlay G. The LAM cell: what is it, where does it come from, and why does it grow? Am J Physiol Lung Cell Mol Physiol 2004; 286: L690-L693.

18 Kwiatkowski DJ. Tuberous sclerosis: from tubers to mTOR. Ann Hum Genet 2003; 67: 87-96.

19 Goncharova EA, Krymskaya VP. Pulmonary lymphangioleiomyomatosis (LAM): progress and current challenges. J Cell Biochem 2008; 103: 369-382.

20 Smolarek TA, Wessner LL, McCormack FX, et al. Evidence that lymphangiomyomatosis is caused by TSC2 mutations: chromosome 16p13 loss of heterozygosity in angiomyolipomas and lymph nodes from women with lymphangiomyomatosis. Am J Hum Genet 1998; 62: 810-815.

21 Vianna FG, Marchiori E, Zanetti G, et al. Tuberous sclerosis with pulmonary lymphangioleiomyomatosis and renal angiomyolipomas. Computed tomographic findings: a case report. Cases J 2009; 2: 9124.

22 Taylor JR, Ryu J, Colby TV, et al. Lymphangioleiomyomatosis. Clinical course in 32 patients. N Engl J Med 1990; 323: $1254-1260$.

23 Almoosa KF, Ryu JH, Mendez J, et al. Management of pneumothorax in lymphangioleiomyomatosis: effects on recurrence and lung transplantation complications. Chest 2006; 129: 1274-1281.

24 Steagall WK, Glasgow CG, Hathaway OM, et al. Genetic and morphologic determinants of pneumothorax in lymphangioleiomyomatosis. Am J Physiol Lung Cell Mol Physiol 2007; 293: L800-L808.

25 Harari S, Torre O, Moss J. Lymphangioleiomyomatosis: what do we know and what are we looking for? Eur Respir Rev 2011; 20: 34-44.

26 Pallisa E, Sanz P, Roman A, et al. Lymphangioleiomyomatosis: pulmonary and abdominal findings with pathologic correlation. Radiographics 2002; 22: S185-S198.

27 Tobino K, Johkoh T, Fujimoto K, et al. Computed tomographic features of lymphangioleiomyomatosis: evaluation in 138 patients. Eur J Radiol 2015; 84: 534-541. 
Avila NA, Dwyer AJ, Rabel A, et al. Sporadic lymphangioleiomyomatosis and tuberous sclerosis complex with lymphangioleiomyomatosis: comparison of CT features. Radiology 2007; 242: 277-285.

Ristagno RL, Biddinger PW, Pina EM, et al. Multifocal micronodular pneumocyte hyperplasia in tuberous sclerosis. AJR Am J Roentgenol 2005; 184: Suppl., S37-S39.

Avila NA, Kelly JA, Chu SC, et al. Lymphangioleiomyomatosis: abdominopelvic CT and US findings. Radiology 2000; 216: 147-153.

Dixon BP, Hulbert JC, Bissler JJ. Tuberous sclerosis complex renal disease. Nephron Exp Nephrol 2011; 118: e15-e20.

Koyama M, Johkoh T, Honda O, et al. Chronic cystic lung disease: diagnostic accuracy of high-resolution CT in 92 patients. AJR Am J Roentgenol 2003; 180: 827-835.

Bonelli FS, Hartman TE, Swensen SJ, et al. Accuracy of high-resolution CT in diagnosing lung diseases. AJR Am J Roentgenol 1998; 170: 1507-1512.

( management of lymphangioleiomyomatosis. Eur Respir J 2010; 35: 14-26.

Seyama K, Kumasaka T, Souma S, et al. Vascular endothelial growth factor-D is increased in serum of patients with lymphangioleiomyomatosis. Lymphat Res Biol 2006; 4: 143-152.

Glasgow CG, Avila NA, Lin JP, et al. Serum vascular endothelial growth factor-D levels in patients with lymphangioleiomyomatosis reflect lymphatic involvement. Chest 2009; 135: 1293-1300.

Urban T, Lazor R, Lacronique J, et al. Pulmonary lymphangioleiomyomatosis. A study of 69 patients. Groupe d'Etudes et de Recherche sur les Maladies “Orphelines” Pulmonaires (GERM”O”P). Medicine 1999; 78: 321-337. Taveira-DaSilva AM, Steagall WK, Moss J. Lymphangioleiomyomatosis. Cancer Control 2006; 13: $276-285$. Radzikowska E. Lymphangioleiomyomatosis: new treatment perspectives. Lung 2015; 193: 467-475.

Taveira-DaSilva AM, Moss J. Management of lymphangioleiomyomatosis. F1000Prime Rep 2014; 6 : 116.

Maurer JR, Ryu J, Beck G, et al. Lung transplantation in the management of patients with lymphangioleiomyomatosis: baseline data from the NHLBI LAM Registry. J Heart Lung Transplant 2007; 26: 1293-1299.

Benden C, Rea F, Behr J, et al. Lung transplantation for lymphangioleiomyomatosis: the European experience. J Heart Lung Transplant 2009; 28: 1-7.

Crausman RS, Jennings CA, Tuder RM, et al. Pulmonary histiocytosis X: pulmonary function and exercise pathophysiology. Am J Respir Crit Care Med 1996; 153: 426-435.

Friedman PJ, Liebow AA, Sokoloff J. Eosinophilic granuloma of lung. Clinical aspects of primary histiocytosis in the adult. Medicine 1981; 60: 385-396.

Vassallo R, Jensen EA, Colby TV, et al. The overlap between respiratory bronchiolitis and desquamative interstitial pneumonia in pulmonary Langerhans cell histiocytosis: high-resolution CT, histologic, and functional correlations. Chest 2003; 124: 1199-1205.

Tazi A, Soler P, Hance AJ. Adult pulmonary Langerhans' cell histiocytosis. Thorax 2000; 55: 405-416.

Vassallo R, Walters PR, Lamont J, et al. Cigarette smoke promotes dendritic cell accumulation in COPD; a Lung Tissue Research Consortium study. Respir Res 2010; 11: 45.

Jawad H, Walker CM, Wu CC, et al. Cystic interstitial lung diseases: recognizing the common and uncommon entities. Curr Probl Diagn Radiol 2014; 43: 115-127.

Kambouchner M, Basset F, Marchal J, et al. Three-dimensional characterization of pathologic lesions in pulmonary langerhans cell histiocytosis. Am J Respir Crit Care Med 2002; 166: 1483-1490.

Abbott GF, Rosado-de-Christenson ML, Franks TJ, et al. From the archives of the AFIP: pulmonary Langerhans cell histiocytosis. Radiographics 2004; 24: 821-841.

Mendez JL, Nadrous HF, Vassallo R, et al. Pneumothorax in pulmonary Langerhans cell histiocytosis. Chest 2004; 125: 1028-1032.

Fartoukh M, Humbert M, Capron F, et al. Severe pulmonary hypertension in histiocytosis X. Am J Respir Crit Care Med 2000; 161: 216-223.

Torre O, Harari S. The diagnosis of cystic lung diseases: a role for bronchoalveolar lavage and transbronchial biopsy? Respir Med 2010; 104: Suppl., S81-S85.

Lorillon G, Bergeron A, Detourmignies L, et al. Cladribine is effective against cystic pulmonary Langerhans cell histiocytosis. Am J Respir Crit Care Med 2012; 186: 930-932.

Lazor R, Etienne-Mastroianni B, Khouatra C, et al. Progressive diffuse pulmonary Langerhans cell histiocytosis improved by cladribine chemotherapy. Thorax 2009; 64: 274-275.

Boehler A, Speich R, Russi EW, et al. Lung transplantation for lymphangioleiomyomatosis. N Engl J Med 1996; 335: $1275-1280$.

Tazi A, Montcelly L, Bergeron A, et al. Relapsing nodular lesions in the course of adult pulmonary Langerhans cell histiocytosis. Am J Respir Crit Care Med 1998; 157: 2007-2010.

Cordier JF, Cottin V, Khouatra C, et al. Multiple cystic lung diseases. In: Cottin V, Cordier JF, Richeld L, eds. Orphan Lung diseases. A Clinical Guide to Rare Lung Disease. London, Springer, 2015.

Kluijt I, de Jong D, Teertstra HJ, et al. Early onset of renal cancer in a family with Birt-Hogg-Dubé syndrome. Clin Genet 2009; 75: 537-543.

Gunji Y, Akiyoshi T, Sato T, et al. Mutations of the Birt Hogg Dube gene in patients with multiple lung cysts and recurrent pneumothorax. J Med Genet 2007; 44: 588-593.

Gupta N, Seyama K, McCormack FX. Pulmonary manifestations of Birt-Hogg-Dubé syndrome. Fam Cancer 2013; 12: 387-396.

Furuya M, Tanaka R, Koga S, et al. Pulmonary cysts of Birt-Hogg-Dube syndrome: a clinicopathologic and immunohistochemical study of 9 families. Am J Surg Pathol 2012; 36: 589-600.

Adley BP, Smith ND, Nayar R, et al. Birt-Hogg-Dubé syndrome: clinicopathologic findings and genetic alterations. Arch Pathol Lab Med 2006; 130: 1865-1870.

Agarwal PP, Gross BH, Holloway BJ, et al. Thoracic CT findings in Birt-Hogg-Dube syndrome. AJR Am J Roentgenol 2011; 196: 349-352.

Tobino K, Hirai T, Johkoh T, et al. Differentiation between Birt-Hogg-Dubé syndrome and lymphangioleiomyomatosis: quantitative analysis of pulmonary cysts on computed tomography of the chest in 66 females. Eur J Radiol 2012; 81: 1340-1346. 
Menko FH, van Steensel MA, Giraud S, et al. Birt-Hogg-Dubé syndrome: diagnosis and management. Lancet Oncol 2009; 10: 1199-1206.

Ayo DS, Aughenbaugh GL, Yi ES, et al. Cystic lung disease in Birt-Hogg-Dube syndrome. Chest 2007; 132: 679-684. Pavlovich CP, Walther MM, Eyler RA, et al. Renal tumors in the Birt-Hogg-Dube syndrome. Am J Surg Pathol 2002; 26: 1542-1552.

Escuissato DL, de Almeida Teixeira BC, Warszwiak D, et al. Renal tumor associated with pulmonary cysts: Birt-Hogg-Dubé syndrome. QJM 2014; 107: 851-852.

Toro JR, Wei MH, Glenn GM, et al. BHD mutations, clinical and molecular genetic investigations of Birt-Hogg-Dube syndrome: a new series of 50 families and a review of published reports. J Med Genet 2008; 45: 321-331.

Toro JR, Pautler SE, Stewart L, et al. Lung cysts, spontaneous pneumothorax, and genetic associations in 89 families with Birt-Hogg-Dube syndrome. Am J Respir Crit Care Med 2007; 175: 1044-1053.

Dal Sasso AA, Belém LC, Zanetti G, et al. Birt-Hogg-Dubé syndrome. State-of-the-art review with emphasis on pulmonary involvement. Respir Med 2015; 109: 289-296.

Swigris JJ, Berry GJ, Raffin TA, et al. Lymphoid interstitial pneumonia: a narrative review. Chest 2002; 122: 2150-2164.

Ichikawa Y, Kinoshita M, Koga T, et al. Lung cyst formation in lymphocytic interstitial pneumonia: CT features. J Comput Assist Tomogr 1994; 18: 745-748.

American Thoracic Society, European Respiratory Society. American Thoracic Society/European Respiratory Society International Multidisciplinary Consensus Classification of the Idiopathic Interstitial Pneumonias. Am J Respir Crit Care Med 2002; 165: 277-304.

Johkoh T, Muller NL, Pickford HA, et al. Lymphocytic interstitial pneumonia: thin-section CT findings in 22 patients. Radiology 1999; 212: 567-572.

Dalvi V, Gonzalez EB, Lovett L. Lymphocytic interstitial pneumonitis (LIP) in Sjögren's syndrome: a case report and a review of the literature. Clin Rheumatol 2007; 26: 1339-1343.

Croda J, Croda MG, Neves A, et al. Benefit of antiretroviral therapy on survival of human immunodeficiency virus-infected patients admitted to an intensive care unit. Crit Care Med 2009; 37: 1605-1611.

Fujii T, Nakamura T, Iwamoto A. Pneumocystis pneumonia in patients with HIV infection: clinical manifestations, laboratory findings, and radiological features. I Infect Chemother 2007; 13: 1-7.

Marchiori E, Müller NL, Soares Souza A Jr, et al. Pulmonary disease in patients with AIDS: high-resolution CT and pathologic findings. AJR Am J Roentgenol 2005; 184: 757-764.

Vogel MN, Vatlach M, Weissgerber P, et al. HRCT-features of Pneumocystis jiroveci pneumonia and their evolution before and after treatment in non-HIV immunocompromised patients. Eur J Radiol 2012; 81: 1315-1320.

Grant LA, Babar J, Griffin N. Cysts, cavities, and honeycombing in multisystem disorders: differential diagnosis and findings on thin-section CT. Clin Radiol 2009; 64: 439-448.

Chong S, Lee KS, Yi CA, et al. Pulmonary fungal infection: imaging findings in immunocompetent and immunocompromised patients. Eur J Radiol 2006; 59: 371-383.

Sakai M, Yamaoka M, Kawaguchi M, et al. Multiple cystic pulmonary amyloidosis. Ann Thorac Surg 2011; 92: e109.

Gertz MA, Dispenzieri A. Immunoglobulin light-chain amyloidosis: growing recognition, new approaches to therapy, active clinical trials. Oncology 2012; 26: 152-161.

Marchiori E, Zanetti G, Hocchegger B, et al. Cystic amyloidosis or lymphoid interstitial pneumonia associated with amyloidosis? A diagnostic challenge. Ann Thorac Surg 2012; 94: 1041-1042.

Jeong YJ, Lee KS, Chung MP, et al. Amyloidosis and lymphoproliferative disease in Sjögren syndrome: thin-section computed tomography findings and histopathologic comparisons. J Comput Assist Tomogr 2004; 28: 776-781.

Ohdama S, Akagawa S, Matsubara O, et al. Primary diffuse alveolar septal amyloidosis with multiple cysts and calcification. Eur Respir J 1996; 9: 1569-1571.

Sheard S, Nicholson AG, Edmunds L, et al. Pulmonary light-chain deposition disease: CT and pathology findings in nine patients. Clin Radiol 2015; 70: 515-522.

Colombat M, Caudroy S, Lagonotte E, et al. Pathomechanisms of cyst formation in pulmonary light chain deposition disease. Eur Respir J 2008; 32: 1399-1403.

Bhargava P, Rushin JM, Rusnock EJ, et al. Pulmonary light chain deposition disease: report of five cases and review of the literature. Am J Surg Pathol 2007; 31: 267-276.

Hoag JB, Sherman M, Fasihuddin Q, et al. A comprehensive review of spontaneous pneumothorax complicating sarcoma. Chest 2010; 138: 510-518.

Somasekharan Nair KK, Zabell AS, Vo KL, et al. Pneumothorax: a classical presentation of metastatic scalp angiosarcoma. Ann Thorac Surg 2012; 94: e77-e78.

Akira M, Yamamoto $\mathrm{S}$, Hara $\mathrm{H}$, et al. Serial computed tomographic evaluation in desquamative interstitial pneumonia. Thorax 1997; 52: 333-337.

Franquet T, Hansell DM, Senbanjo T, et al. Lung cysts in subacute hypersensitivity pneumonitis. J Comput Assist Tomogr 2003; 27: 475-478.

Ryu JH, Myers JL, Swensen SJ. Bronchiolar disorders. Am J Respir Crit Care Med 2003; 168: 1277-1292.

Karpman C, Aughenbaugh GL, Ryu JH. Pneumothorax and bullae in Marfan syndrome. Respiration 2011; 82: 219-224.

Irion KL, Gasparetto TD, Marchiori E, et al. Neurofibromatosis type 1 with tracheobronchial neurofibromas: case report with emphasis on tomographic findings. J Thorac Imaging 2008; 23: 194-196.

Zamora AC, Collard HR, Wolters PJ, et al. Neurofibromatosis-associated lung disease: a case series and literature review. Eur Respir J 2007; 29: 210-214.

Dowton SB, Pincott S, Demmer L. Respiratory complications of Ehlers-Danlos syndrome type IV. Clin Genet 1996; 50: 510-514.

Irion KL, Hocchegger B, Marchiori E, et al. Proteus syndrome: high-resolution CT and CT pulmonary densitovolumetry findings. $J$ Thorac Imaging 2009; 24: 45-48.

02 Thompson GR 3rd. Pulmonary coccidioidomycosis. Semin Respir Crit Care Med 2011; 32: 754-763. 
103 Capone D, Marchiori E, Wanke B, et al. Acute pulmonary coccidioidomycosis: CT findings from 15 patients. Br J Radiol 2008; 81: 721-724.

104 Costa AN, Marchiori E, Benard G, et al. Lung cysts in chronic paracoccidioidomycosis. J Bras Pneumol 2013; 39: 368-372.

105 Marchiori E, Araujo Neto C, Meirelles GS, et al. Laryngotracheobronchial papillomatosis: findings on computed tomography scans of the chest. J Bras Pneumol 2008; 34: 1084-1089.

106 Marchiori E, Zanetti G, Barreto MM, et al. Recurrent respiratory papillomatosis with malignant transformation. Respirology 2010; 15: 726-728.

107 Marchiori E, Zanetti G, Mauro, et al. Tracheobronchial papillomatosis with diffuse cavitary lung lesions. Pediatr Radiol 2010; 40: 1301-1132.

108 Foster WL Jr, Gimenez EI, Roubidoux MA, et al. The emphysemas: radiologic-pathologic correlations. Radiographics 1993; 13: 311-328.

109 Marchiori E, Canella C, Hochhegger B, et al. An uncommon complication of staphylococcal pneumonia: pneumopericardium with cardiac tamponade. Thorax 2015; 70: 395. 